\title{
Data Item
}

National Cancer Institute

\section{Source}

National Cancer Institute. Data Item. NCI Thesaurus. Code C142479.

A named component of a data element, usually the smallest component. (ANSI) 